\section{A new year}

A $\mathrm{s}$ we begin a new year, it is always important to reflect on what has been achieved, and especially to acknowledge the efforts of all those who have made the success possible. In this issue, we are publishing the names of all individuals who have served as reviewers for the Journal during 2006. Importantly, many of these individuals have performed several reviews for the Journal during the year, and I recognize that most also perform reviews for other journals. We are always striving to increase out database of reviewers and would appreciate any help that you can provide. If you are not currently in our database, but would like to be added, please let us know. On the other hand, if you know someone who would be a good reviewer, also please let us know. To help with this process, we will soon be asking department heads, division leaders and others to help us with expanding our reviewer base. Doing journal reviews can be a rewarding experience, and for those with research fellows and trainees working with them, sharing in a review can be an extraordinarily good teaching and learning experience.

One of the major reasons for wanting to expand our reviewer base is the continual struggle in the editorial office to obtain reviews in a timely manner. We ask our reviewers to commit to providing the review within two to three weeks, but the median time from receipt of the paper until we can offer the authors our initial decision continues to be approximately one month. Clearly, not all of the delay is caused by the reviewers - editors do accept responsibility for some of the time lost. Our median time to decision compares well with those of most other journals, but authors continue to be frustrated by these delays. Of course, this delay is added to that which occurs from the time of decision to publication.

We want to recognize those individuals who make that extra effort to complete their reviews quickly. On the following page is a list of the 10 fastest reviews/reviewers during 2006. Our sincere appreciation is extended to these special people.

We have also made some minor revisions to the Instructions to Authors, which can be accessed at $<$ www.pulsus.com/cardiol/instruct.htm>. The most important change is that beginning immediately, the number of references for Case Reports will be limited to five. This means that Case Reports will no longer include a 'review of the literature', although reference to a few key articles on the subject is encouraged.

On behalf of Pulsus Group Inc, the editorial office and especially Peggy Randall, I take this opportunity to wish everyone a very happy and successful 2007.

Eldon R Smith Editor-in-Chief

\section{À l'aube d'une nouvelle année}

À l'aube d'une nouvelle année, il est toujours Aimportant de réfléchir aux les réalisations passées et, surtout, de reconnaître les efforts de ceux et celles qui ont rendu ces réalisations possibles. C'est pourquoi nous publions, dans le présent numéro, le nom de tous les examinateurs et examinatrices qui ont contribué au succès du Journal en 2006. Point important à souligner, bon nombre d'entre eux ont effectué plusieurs examens pour le Journal au cours de l'année, sans compter que la plupart en font pour d'autres revues également. Nous sommes toujours à la recherche d'examinateurs et d'examinatrices, et votre aide serait grandement appréciée. Si vous n'êtes pas inscrit dans la banque de données à ce titre et que vous aimeriez l'être, ou encore si vous connaissez quelqu'un qui ferait un bon examinateur, faites-le-nous savoir. De plus, nous solliciterons bientôt des chefs de service, des responsables de division et d'autres personnes pour nous aider à allonger la liste d'examinateurs. Réviser des articles pour une revue peut se révéler une expérience gratifiante, tout en étant une excellente expérience d'enseignement pour les examinateurs et d'apprentissage pour les chercheurs et les stagiaires qui gravitent autour d'eux.

Une des raisons pour lesquelles nous voulons avoir plus d'examinateurs est la lutte que doit livrer constamment l'équipe de rédaction pour remettre les articles jugés le plus rapidement possible. Nous demandons aux examinateurs et examinatrices de renvoyer les articles dans un délai de deux à trois semaines, mais le temps médian qui s'écoule entre le moment de la réception de l'article et le moment de la communication de la décision se maintient à environ un mois. Certes, on ne peut imputer aux seuls examinateurs les retards de traitement; l'équipe de rédaction a aussi sa part de responsabilité. Il est vrai que le temps médian écoulé jusqu'au moment de la décision se compare tout à fait à celui observé dans la plupart des autres revues, mais les délais constituent toujours une source d'irritation pour les auteurs et auteures. Évidemment, ce délai s'ajoute à celui qui est prévu entre le moment de la décision et le moment de la publication.

Nous tenons à remercier ceux et celles qui ont fait un effort supplémentaire, cette année, pour remettre rapidement les résultats de leur analyse. Suit un peu plus loin la liste des dix examinateurs et examinatrices les plus rapides en 2006. Nous leur transmettons nos plus sincères remerciements.

Par ailleurs, nous avons apporté quelques petites modifications aux instructions données aux auteurs, accessibles à $<$ www.pulsus.com/cardiol/instruct.htm $>$. La plus importante est la limitation à cinq du nombre de références dans les exposés de cas, et ce, dès maintenant. Cela veut dire que désormais les exposés ne comprendront plus un « examen de la documentation ", bien qu'on encourage les renvois à quelques grands articles de fond sur le sujet. 
During 2006, the following individuals completed a review within one day of the request. What an outstanding achievement - we appreciate it!
Jafna Cox
Hank Duff
Paul Fedak
David Fedida
George Fodor
Marvin Fritzler
Jean Gray
Kathryn King
Andrew Maitland
Gary Newton
Fred Ofosu
Tim Pollak
Christopher Simpson
David Spence
Henk ter Keurs
John Veinot
Subodh Verma
David Waters
Lisa Welikovitch

Enfin, au nom de Pulsus Group, de l'équipe de rédaction et, en particulier, de Peggy Rendall, je profite de l'occasion pour souhaiter à chacun et à chacune d'entre vous une bonne année 2007, remplie de joie et de succès.

Eldon R Smith Rédacteur en chef

Voici le nom des examinateurs et des examinatrices qui ont remis leur analyse une journée plus tôt que prévu au cours de l'année 2006. Tout un exploit ! Merci beaucoup !
Jafna Cox
Hank Duff
Paul Fedak
David Fedida
George Fodor
Marvin Fritzler
Jean Gray
Kathryn King
Andrew Maitland
Gary Newton
Fred Ofosu
Tim Pollak
Christopher Simpson
David Spence
Henk ter Keurs
John Veinot
Subodh Verma
David Waters
Lisa Welikovitch

The views expressed on this page are those of the Editor-in-Chief and are not intended to reflect the opinions of the Canadian Cardiovascular Society or Pulsus Group Inc.
Le présent point de vue n'engage que le rédacteur en chef et ne reflète pas nécessairement celui de la Société canadienne de cardiologie ou de Pulsus Group Inc. 